\title{
Miniaturized Impedance Measurement System for Battery Diagnosis
}

Uwe Tröltzsch, Olfa Kanoun

Professur für Mess- und Sensortechnik

Technische Universität Chemnitz, D-09107 Chemnitz

Email: mst@tu-chemnitz.de

\begin{abstract}
Battery diagnosis information is required for many applications. Relevant quantities are state of charge (SOC), ampere hour capacity and internal resistance. Impedance spectroscopy is a preferred method for battery diagnosis because of its ability to separate individual mechanisms due to their different frequency behavior. A prototype for a miniaturized low-cost impedance measurement system was realized especially for battery diagnosis. For impedance measurement a digital signal processing method was used. A sinusoidal signal was used as excitation signal. Special care was dedicated to the correction of parasitic effects due to parasitic wire capacitances and the parasitic capacitance of discrete circuit elements. The developed system is compact and can be integrated in different applications.
\end{abstract}

\section{Introduction}

Reliable operation of batteries requires assured diagnosis information. Several methods like charge balancing, impedance measurement and impedance spectroscopy are available [1]. Impedance spectroscopy is very promising because of being able of extracting spectrally distributed information [2]. For a wide practical application a suitable measurement system is required having properties such as low cost realization, data bus connection and compact dimensions. For this purpose, a special measurement system was developed. The main focus thereby is devoted to a realizing good signal quality for the characterization of aging behavior in spite of limited resources.

\section{Measuring battery aging}

Aging mechanisms of batteries have a negative influence on the electrical behavior like ampere hour capacity, self discharge rate and inner resistance. The large amount of available methods for determining these quantities shows the importance [1, 2, 3]. Charge balancing based on current measurements is widely used for determining actual state of charge (SOC) and battery capacity. In this case measurement results allow predicting the battery behavior for a short time but reasons for decreasing performance cannot be explained.

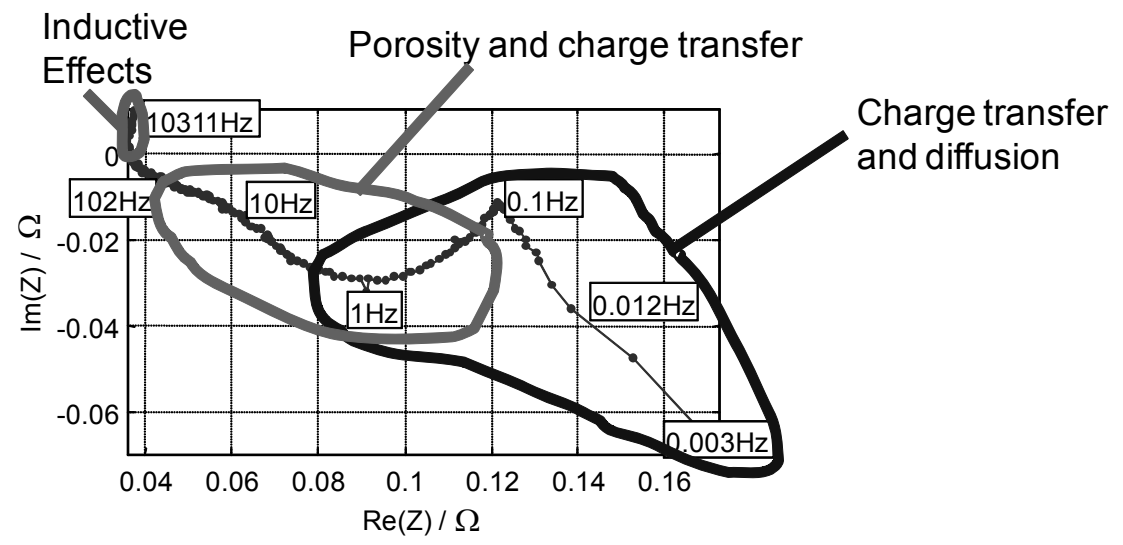

Figure 1: Separating several electrochemical mechanisms within a lithium ion battery using impedance spectroscopy 
Impedance spectroscopy uses the different frequency behavior of individual mechanisms for their separation (s. Figure 1). It allows a physical interpretation of observed changes of the inner resistance because of aging mechanisms. Generally the measured spectrum is dependent simultaneously on the battery ampere hour capacity and the SOC. Therefore it is difficult to determine all these quantities [3, 4]. An interesting approach is to combine both charge balancing and impedance spectroscopy. Impedance spectroscopy determines inner resistance, gives a good short time prediction and helps identifying individual mechanisms within the battery. By charge balancing the SOC can be determined. In this paper we consider batteries at a given SOC, because we focus just on aging effects.

\section{Modeling of aging mechanisms}

For modeling aging mechanisms empirical-mathematical and physical-chemical models can be used. Digital Filters, expert systems like fuzzy logic and neural networks or statistical methods like classifiers and the multivariate analysis are examples for empirical-mathematical methods. A physical explanation for the observed behavior is thereby impossible. The results are strongly dependent on the representativeness of the underlying data.

Physical-chemical models allow identifying reasons of decreasing electrical performance because of aging phenomena. The difficulty thereby is to find a model describing all individual mechanism and getting along with few unknown parameters. For interpretation of impedance spectra model parameters are to be determined by nonlinear optimization. For models with a high degree of nonlinearity and a big number of unknown parameters it is difficult to avoid ambiguities during parameter extraction procedure.

\section{Measuring impedance spectra}

For measurement of impedance generally an electrical excitation signal is applied to the device under test (DUT) and the corresponding electrical response is measured. From both signals the impedance can be calculated. Designing the necessary electrical measurement circuit requires choosing adequate signals and algorithms. Fundamental aspects of circuit design, signal and algorithmic are discussed in the following sections.

\subsection{Choosing test signals}

Criteria for choosing a certain excitation signal are:

- Short measurement time,

- Adequate signal to noise ratio,

- Sufficient frequency band.

The decision for one special signal is always a trade off. Low measurement frequencies are only possible with long measurement times and achieve a better signal to noise ratio. In [5] several time continuous signals were compared regarding their efficiency in measuring impedance spectra. Multi sine signals with logarithmically spaced frequencies achieved best results because of equidistant information distribution in the frequency domain and lowest noise level. With pure digital circuits, analog multi sine signals are not be realizable. In this case rectangular pulses with randomly distributed pulse width (maximum length sequences) are an adequate choice. Analog pulse signals are also suitable for impedance measurement. Good results were achieved by using rectangular pulses, si pulses $(\sin (x) / x)$ and Gaussian pulses [6]. The first derivation of these pulses is also interesting for impedance measurement because they have no mean value and thereby do not change the SOC of a battery.

\subsection{Signal acquisition and impedance calculation}

For signal acquisition and impedance calculation digital and analog methods are used (s. Figure 5). Digital signal processing methods are used at low frequencies from $\mu \mathrm{Hz}$ to several $\mathrm{MHz}$ and are applicable for sinusoidal and all other kinds of test signals. For calculating the impedance the fast Fourier transform is used in most cases. The sine fitting (sinfit) method delivers equivalent results in case of well known frequency [7]. 
Digital Signal Processing Method

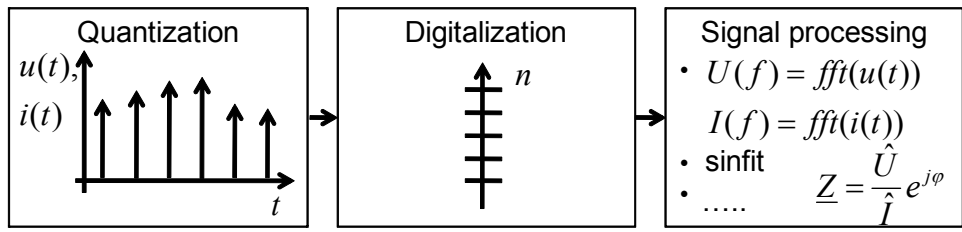

Analog Signal Processing Method

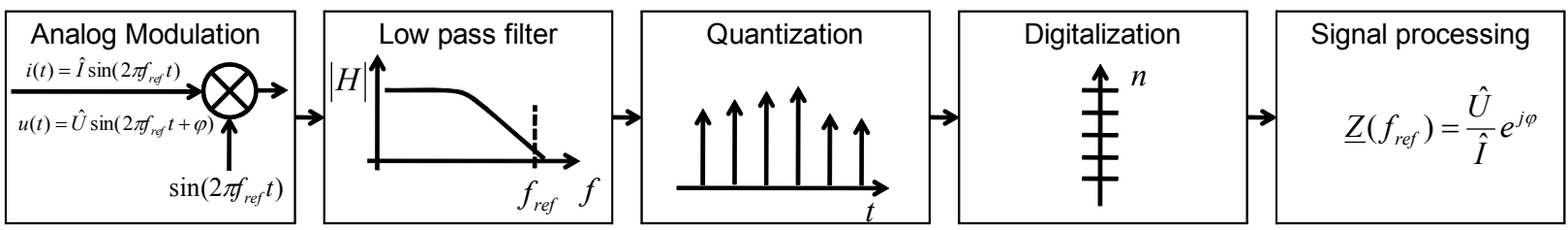

Figure 2: Digital and analog method for impedance measurement

Analog signal processing methods are used at higher frequencies up to several $\mathrm{GHz}$. The slowly changing outputs of the low pass filters can be easily converted with simple analog to digital converters. The impedance is calculated in the digital domain.

\section{Principles and components of the measurement system}

For battery diagnosis only low frequencies are required. Therefore a digital signal processing method is suitable. Because of the high signal quality a multi sine signal is preferred as test signal.

By carefully selecting a microprocessor having a periphery including analog to digital converter and digital to analog converter, bus interface and a powerful arithmetic processing unit a digital method can be implemented at low costs with a small circuit. Except a digital to analog converter all functionalities are available on the selected microprocessor. Using a DDS circuit instead of a digital to analog converter for signal generation is an interim solution. The DDS circuit does not allow generating multi sine signals but sinusoidal, rectangular and triangle signals. The microprocessor contains a powerful DSP-core for calculating tourier transform, sine fit algorithm and other matrix operations. Parameters of simple equivalent circuits can be calculated by using nonlinear optimization.

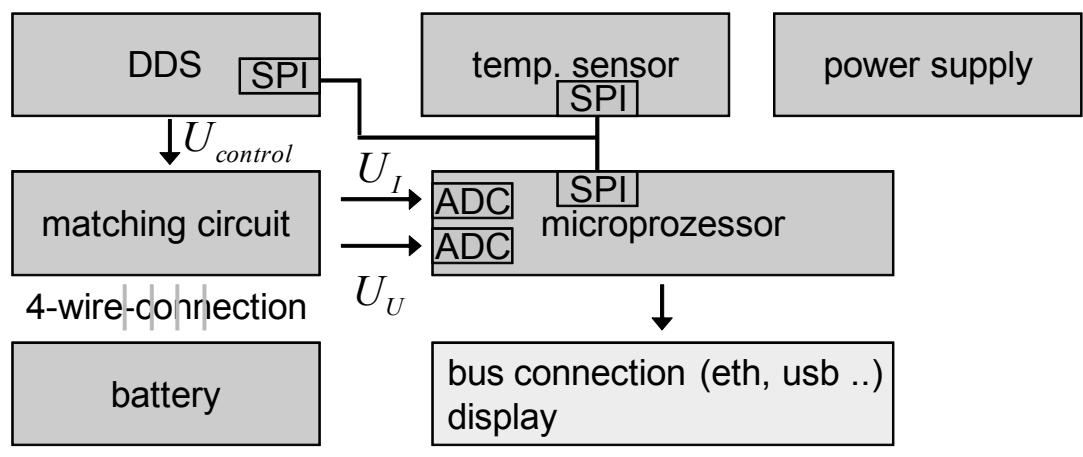

Figure 3: Prototype system design

The proposed measurement system uses commercially available, standardized integrated circuits. The microcontroller controls the measurement system, measures signals and calculates results. An analog matching circuit adapts the signals to the investigated DUT. The measurement system allows measuring typical battery impedances in the range of milliohm at offset voltages up to $12 \mathrm{~V}$. 


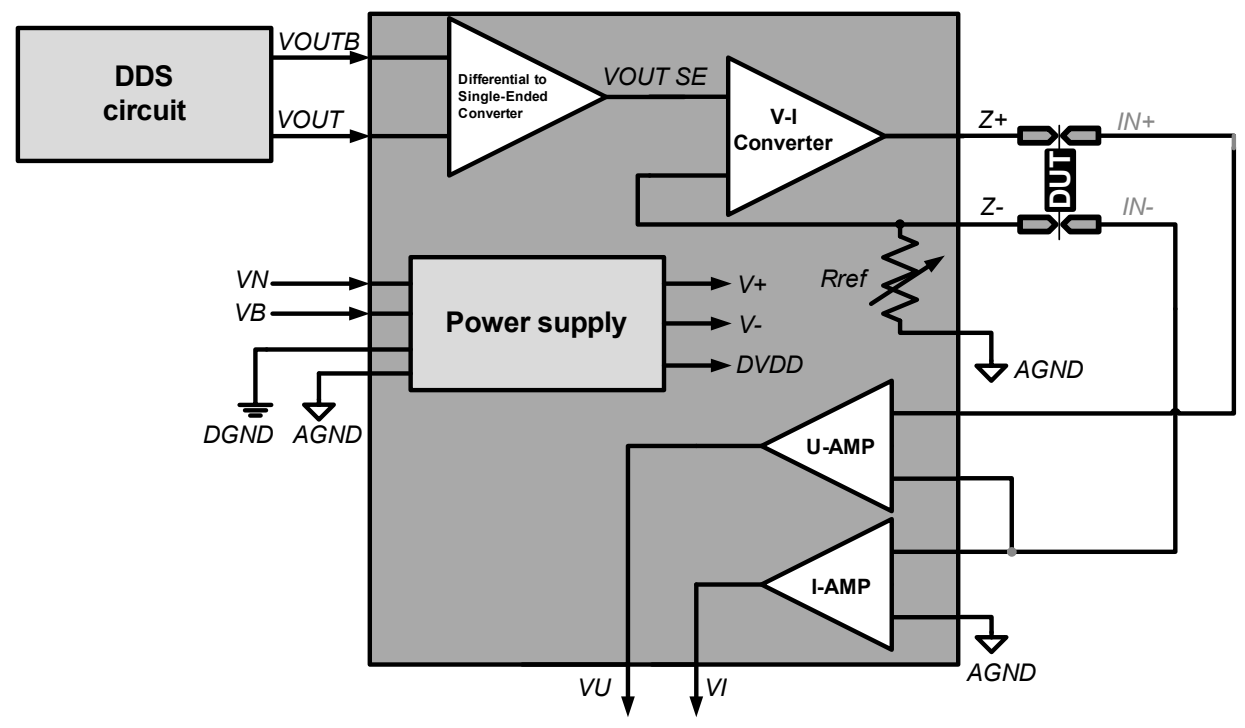

Figure 4: Block diagram matching network [8]

The analog matching network according to figure 5 generates a current which is applied to the DUT. A powerful operational amplifier is working as voltage to current converter. The required voltage is generated by a DDS circuit. Other signal sources like the output of a digital to analog converter are also possible. The current through the DUT and the resulting voltage are measured. The ranges of the voltages at the DUT and at the reference resistor are adapted to the input range of the analog to digital converter using operational amplifiers.

\section{Excitation and signal conditioning}

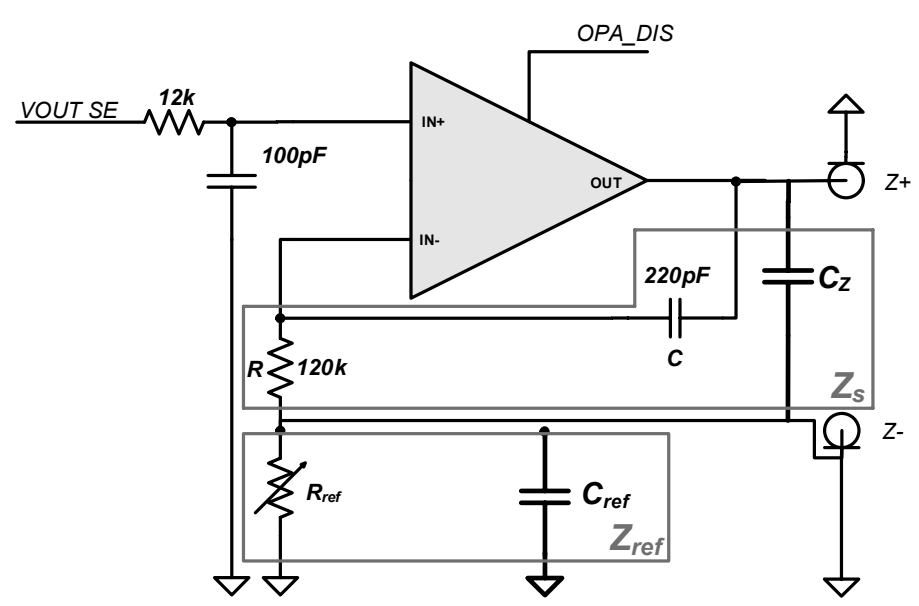

Figure 5: Circuit excitation circuit

Figure 10 shows the signal conditioning circuit. The input voltage gives a set point to the regulator circuit consisting of the operational amplifier. The voltage at the reference resistor follows the input voltage. The resulting current flows through the DUT and through the reference resistor. Currents not only flow through discrete elements but also through parasitic capacitances. Figure 10 also shows parasitic wire capacitances $C_{Z}$ and the parasitic capacitance of the reference resistor $C_{\text {ref. }}$. For a precise phase angle measurement considering these parasitic elements is essential. Especially at higher frequencies a significant current flows through the parasitic elements. The discrete elements $R$ and $C$ are required for stabilization of the amplifier. Oscillations are suppressed. The elements have to be considered for impedance calculation. The DUT is connected by a 4 wire shielded method decreasing disturbing ohmic wire influences. 


\section{Correction algorithms and impedance calculation}

The impedance is calculated from the digitized outputs of the voltage and current amplifier. In advance to the impedance calculation the gains of the voltage and current amplifiers is required to be corrected. After gain correction the voltages at the nodes $I N+$ and $I N$ - are available (s. Figure 5). The formula for impedance calculation is derived from figure 6 . The ratio of the voltages is equal to the ratio of the impedances $Z_{s} \| Z_{\text {DUT }}$ and $Z_{\text {ref }}$ because the same current flows through the impedances.

$$
\frac{I N_{+}-I N_{-}}{I N_{-}}=\frac{Z_{D U T} \cdot Z_{S}}{Z_{D U T}+Z_{S}} \cdot \frac{1}{Z_{r e f}}
$$

Solving equation (1) for the unknown impedance of the DUT $Z_{\text {DUT }}$ gives equation (2). All disturbing influences considered with the circuit according to figure 6 can now be corrected.

$$
Z_{D U T}=\left[\frac{I N_{+}-I N_{-}}{I N_{-}} Z_{r e f}\right] \cdot \frac{1}{1-\frac{\left[\frac{I N_{+}-I N_{-}}{I N_{-}} Z_{r e f}\right]}{Z_{S}}}
$$

The first part of equation (2) is the well known formula for calculating the impedance from voltages at the DUT and a known reference resistor. The second part is a correction term considering parasitic elements. Its influence increases with decreasing impedance $Z_{S}$ for example by a parallel capacitor to the unknown impedance $Z_{\text {DUT }}$. The quality of the data correction algorithm is assessed by comparing measurement data with calibration data obtained e. g. by an Agilent 4294A impedance analyzer. The corrected data shows a better agreement with the data obtained with the impedance analyzer than raw data (s. Figure 7 ). Especially at high frequencies data correction is indispensable.

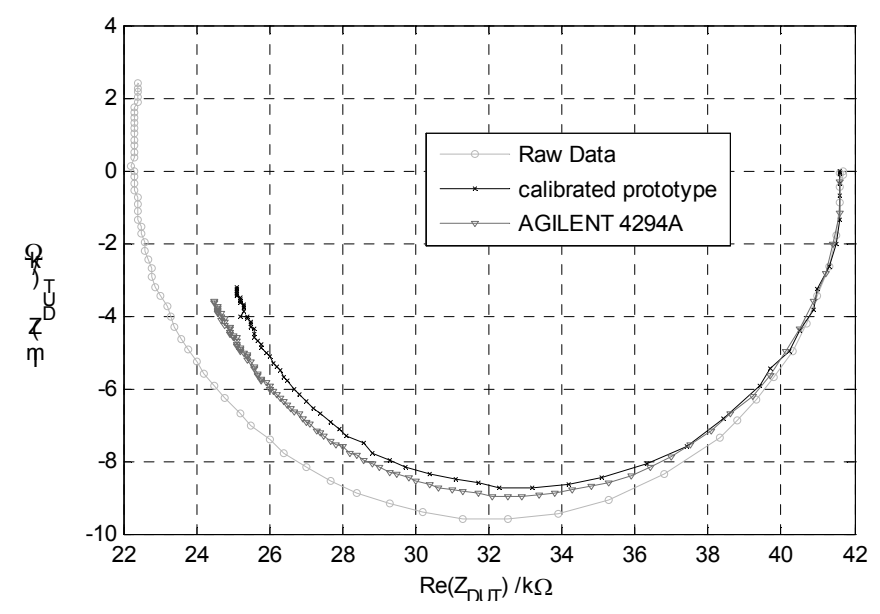

Figure 6: Assessing the quality of the correction algorithm

\section{Experimental Results}

Figure 8 shows impedance data obtained for a lithium ion cell. All mechanisms relevant for battery diagnosis are visible. At high frequencies inductive effects are dominating, at central frequencies the charge transfer reaction is visible and at low frequencies diffusion is observed [9]. 

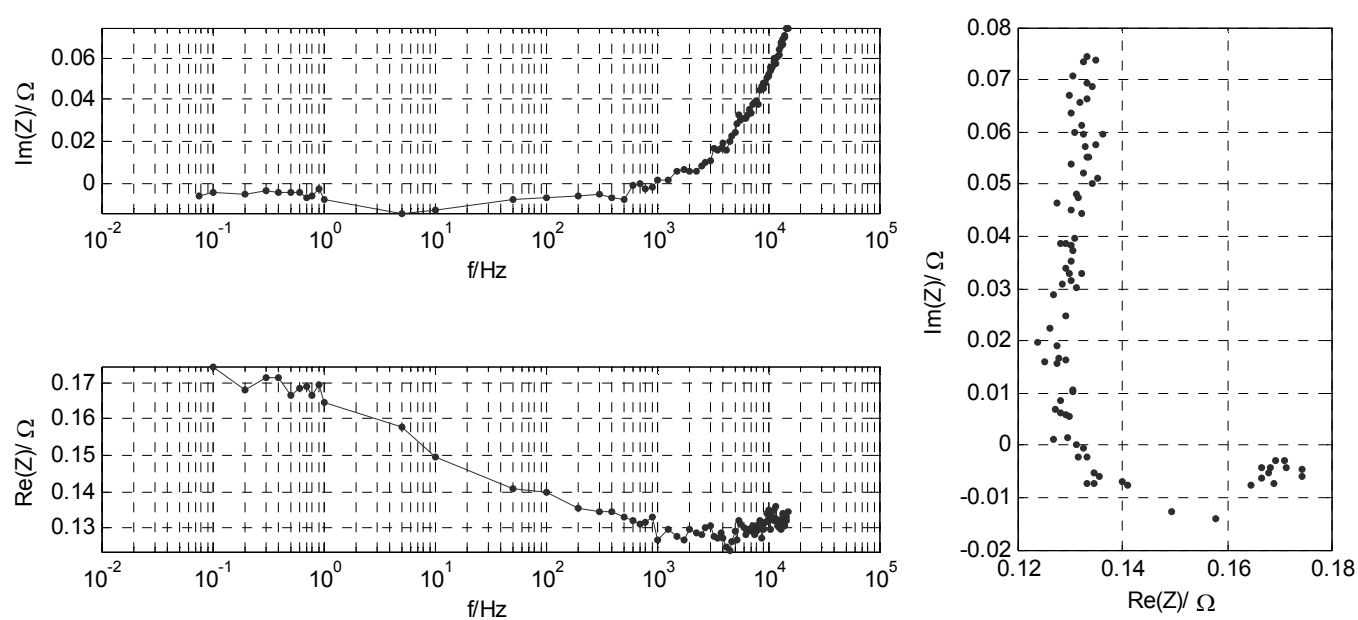

Figure 7: Impedance spectrum of a lithium ion battery obtained with the prototype

\section{Conclusion}

A prototype for a miniaturized low-cost impedance measurement system was realized especially for battery diagnosis. All functionalities like impedance measurement, calculation of impedance spectrum, calculation of simple physical model and deriving diagnosis information can be provided. The measurement system can be integrated in several applications, such as automotive and health care. Further developments will consider a fully digital signal generation, the use of digitally programmable amplifiers and the extension of the voltage range. The implementation of diagnosis algorithms is a big challenge. Advanced methods, like nonlinear parameter extraction and mathematical methods, profit from calculation power of microprocessors. Implementing these methods within the measurement system is the next step.

\section{References}

[1] V. Pop, H.J. Bergveld, P.H.L. Notten, and P.P.L. Regtien. State-of-the-art of battery stateof-charge determination. Measurement Science and Technology, 16(12), pp. R93-R110, 2005.

[2] S. Piller, M. Perrin, and A. Jossen. Methods for state-of-charge determination and their applications. Journal of Power Sources, 96(1), pp.113-120, 2001.

[3] F. Huet. A review of impedance measurements for determination of the state-of-charge or state-of-health of secondary batteries. Journal of Power Sources, 70(1), pp. 59-69, 1998.

[4] S. Rodrigues, N. Munichandraiah, and A. K. Shukla. A review of state-of-charge indication of batteries by means of ac impedance measurements. Journal of Power Sources, 87(12), pp. 12-20, 2000.

[5] G. S. Popkirov and R. N. Schindler. A new impedance spectrometer for the investigation of electrochemical systems. Review of Scientific Instrument, 63(11), pp. 5366-5372, 1992.

[6] M. Min, Pliquett U., T. Nacke, A. Barthel, P. Annus, and R. Land. Signals in bioimpedance measurement: Different waveforms for different tasks. In 13th International Conference on Electrical Bioimpedance and the 8th Conference on Electrical Impedance Tomography, volume 17, pp. 181-184, Graz, Austria, 29 August - 2 September 2007.

[7] T. Radil, P.M. Ramos, and A.C. Serra. Impedance measurement with sine-fitting algorithms implemented in a dsp portable device. IEEE Transactions on Instrumentation and Measurement, 57(1), pp. 197-204, 2008.

[8] A. Kurz. Portables messsystem zur impedanzmessung. Master's thesis, Professur für Mess- und Sensortechnik, Technische Universität Chemnitz, 2008.

[9] U. Tröltzsch. Modellbasierte Zustandsdiagnose von Gerätebatterien. Nr. 1088 in Fortschritt-Berichte Reihe 8. VDI Verlag, Düsseldorf, 2006. 